Phase III of solid methane: The orientational potential and rotational tunneling

Alfred Hüller, Michael Prager, Werner Press, and Tilo Seydel

Citation: The Journal of Chemical Physics 128, 034503 (2008); doi: 10.1063/1.2822293

View online: https://doi.org/10.1063/1.2822293

View Table of Contents: http://aip.scitation.org/toc/jcp/128/3

Published by the American Institute of Physics

PHYSICS TODAY

WHITEPAPERS
ADVANCED LIGHT CURE ADHESIVES

READ NOW

Take a closer look at what these environmentally friendly adhesive systems can do
PRESENTED BY (8) MASTERBOND 


\title{
Phase III of solid methane: The orientational potential and rotational tunneling
}

\author{
Alfred Hüller \\ Aicherstrasse 9, D-82256 Fürstenfeldbruck, Germany and Theory Group, Institut Laue-Langevin, \\ 6 rue Jules Horowitz, F-38042 Grenoble Cedex 9, France \\ Michael Prager ${ }^{\mathrm{a})}$ \\ Institüt für Festkörperforschung, Forschungszentrum Jülich, D-52425 Jülich, Germany \\ Werner Press \\ Institut für Experimentelle und Angewandte Physik, Universität Kiel, Leibnizstr. 11-19, \\ D-24098 Kiel, Germany \\ Tilo Seydel \\ Institut Laue-Langevin, 6 rue Jules Horowitz, F-38042 Grenoble Cedex 9, France
}

(Received 9 October 2007; accepted 14 November 2007; published online 15 January 2008)

\begin{abstract}
After many unsuccessful efforts, the structure of solid $\mathrm{CD}_{4}$ III finally has been solved. In this paper, we examine if the known tunneling spectra are consistent with the orientational potentials at the two sites with different symmetries. To this end, we study the rotational kinetic energy of the molecules, construct appropriate pocket states for the tunneling problem, and set up a model potential. Approximate energy levels are obtained from the Ritz variational principle. The agreement between the experimentally determined tunneling frequencies and the calculations is rather good, corroborating the findings of the structural analysis. A continuation of this paper will deal with the partly deuterated methanes. (C) 2008 American Institute of Physics. [DOI: 10.1063/1.2822293]
\end{abstract}

\section{INTRODUCTION}

Coherent tunneling of a single atom in a double minimum potential, i.e., the tunneling through the barrier between two equivalent potential pockets, is one of the standard problems in a beginners course of quantum mechanics. Yet, when you look for cases of single atom tunneling in solid state physics, where this knowledge is applicable, then hydrogen in metals is about the only example you find. ${ }^{1,2}$

The reason is that in solids, the distances between equivalent sites typically are a few angstroms, while the potential differences (i.e., energy barriers) are measured on the $\mathrm{eV}$ energy scale. Consequently, the tunneling splitting $\hbar \omega_{t}$ normally amounts to a few $\mu \mathrm{eV}$ at most. Any lattice imperfection, in the vicinity of the double well, might spoil the symmetry and render the pockets inequivalent. The energy asymmetry $\hbar \omega_{a}$ may easily exceed $\hbar \omega_{t}$, i.e., $10^{-4}$ of the barrier height. Then, the atom becomes localized and tunneling is suppressed.

This is, however, rather different in the rotational tunneling of highly symmetric molecules. ${ }^{3-5}$ It differs from single atom tunneling in an important aspect: Groups of atoms exchange places. The orientations of minimum potential energy are related to each other by an exchange of identical particles. These particles are indistinguishable and so are the corresponding orientations. There is no agent that might destroy the symmetry-the pockets are identical under all circumstances. The asymmetry $\hbar \omega_{a}$ is zero identically, and therefore the tunneling in the $\mu \mathrm{eV}$ regime is observable.

\footnotetext{
${ }^{a)}$ Electronic mail: m.prager@fz-juelich.de.
}

There is yet another difference between single atom tunneling and rotational tunneling: The atom distorts the lattice and the distortion is different in the two positions. The atom drags this distortion around, as it moves, and it thus acquires an effective mass, larger than its bare mass. This greatly reduces the splitting $\hbar \omega_{t}$ which depends exponentially on the mass. In rotational tunneling of $\mathrm{CH}_{4}$, also this effect is absent, but it comes back for partly deuterated molecules, e.g., $\mathrm{CH}_{3} \mathrm{D}$.

The examples for rotational tunneling can be classified according to the symmetry of the molecule under study. Famous is $\mathrm{H}_{2},{ }^{6,7}$ where the enormous energy difference between ortho- and parahydrogen is a consequence of the exchange symmetry of the two hydrogen atoms. Methyl groups or ammonia ions are examples for one-dimensional rotations around a single axis in a potential with a threefold symmetry. Here, we are concerned with the rotations of methane molecules in a three-dimensional space of angles. In this space, there are 12 equivalent potential minima or pockets.

Quaternions $^{8-11}$ are a very efficient tool for the formulation of three-dimensional rotations. We shall use them very extensively. Therefore, some of their properties, as far as these are needed in this paper, shall be recapitulated in Sec. II.

The operator of the rotational kinetic energy for a spherical top, e.g., $\mathrm{CH}_{4}$ in terms of quaternions is well known. Its generalization ${ }^{12}$ to symmetric tops $\left(\mathrm{CH}_{3} \mathrm{D}\right.$ or $\left.\mathrm{CHD}_{3}\right)$ or an asymmetric top $\left(\mathrm{CH}_{2} \mathrm{D}_{2}\right)$ will be given in Sec. III.

The determination of the rotational ground state tunnel splitting of molecules in strong orientational potentials is a notoriously difficult problem. For the highly symmetric 
problem of a tetrahedral $\mathrm{XH}_{4}$ or $\mathrm{XD}_{4}$ molecule, in a potential of tetrahedral symmetry, calculations with increasing sophistication $^{13-16}$ have, some time ago, produced reliable values for the tunnel splitting.

If the molecular symmetry and the site symmetry are low, as it is the case for the partially deuterated molecules in phase III of methane: ${ }^{17}$ The problem is even more difficult. Therefore, we were forced to use the pocket states which have nonzero amplitude only in one pocket. ${ }^{16}$ They are well adapted to the problem and their employment is essential for our analysis. The advantages of this method will be recapitulated in Sec. IV. For pedagogical reasons, the method will first be explained for the much simpler case of onedimensional methyl rotation in Sec. IV A and then be applied to three-dimensional rotations in Sec. IV B. The Ritz variational principle will be used to calculate the tunneling splitting from the energy differences in the ground state multiplet. As these differences are extremely small, the Ritz method necessitates very good test wave functions. These are introduced in Sec. V. It is rather amazing that a simple $r^{-n}$ atom-atom potential explains the experimental findings for the orientational order of the molecules in phase III rather well. As long as $n$ is larger than 2.2, its precise value is rather unimportant. This is demonstrated in Sec. VI. The same interaction, supplemented by a strength factor, is used in Sec. VII for the determination of the tunneling frequencies in the orientational potential.

\section{QUATERNIONS}

In the following, we use two alternative ways to denote quaternions, ${ }^{8-11}$ either by

$$
\tau=\left(\tau_{1}, \tau_{2}, \tau_{3}, \tau_{4}\right)
$$

or by

$$
\tau=\left(i \tau_{1}+j \tau_{2}+k \tau_{3}+\tau_{4}\right)
$$

with the multiplication rules

$$
i i=j j=k k=-1
$$

and

$$
\begin{aligned}
& i j=-j i=k, \\
& j k=-k j=i, \\
& k i=-i k=j .
\end{aligned}
$$

Quaternions of unit length, with

$$
\sum_{i=1}^{4} \tau_{i}^{2}=1
$$

are very convenient for the description of the rotations of a rigid body. Here, we mention only those of their properties which will be needed in the following. For example, we do not need and mention their relation to the Euler angles. For a more comprehensive account, we refer our readers to Refs. 10 and 11.

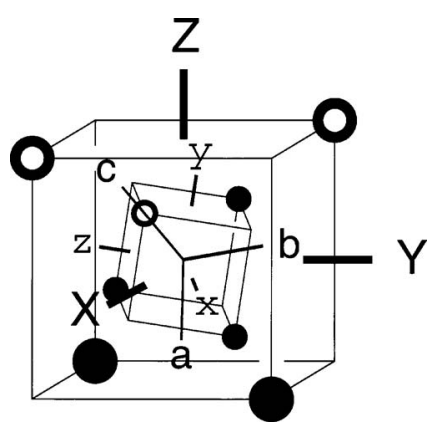

FIG. 1. $\mathrm{A} \mathrm{CHD}_{3}$ or $\mathrm{CH}_{3} \mathrm{D}$ tetrahedron in a surrounding with a twofold symmetry. The $(X, Y, Z)$ coordinate system is fixed in the crystal and the $Z$ axis is the twofold rotation axis. In the $(x, y, z)$ coordinate system, the molecule is in its standard orientation and the axes of the $(a, b, c)$ system are parallel to the principal axes of the moment of inertia.

The rotation of the body by an angle $\gamma$ around an axis parallel to the unit vector $\mathbf{e}=\left(e_{1}, e_{2}, e_{3}\right)$ is described by the quaternion

$$
\tau=\left(\tau_{1}, \tau_{2}, \tau_{3}, \tau_{4}\right)=\left(e_{1} \sin \frac{\gamma}{2}, e_{2} \sin \frac{\gamma}{2}, e_{3} \sin \frac{\gamma}{2}, \cos \frac{\gamma}{2}\right)
$$

From this relation, it is immediately clear that $\tau$ $=\left(\tau_{1}, \tau_{2}, \tau_{3}, \tau_{4}\right)$ and its antipodal point $-\tau=\left(-\tau_{1},-\tau_{2},-\tau_{3}\right.$, $-\tau_{4}$ ) on the four-dimensional unit sphere indicate the same rotation of the rigid body. The latter denotes a rotation around $\mathbf{e}$ by the angle $2 \pi+\gamma$, i.e., also by $\gamma$. Note that $\sin ((2 \pi+\gamma) / 2)=-\sin (\gamma / 2)$ and also that $\cos ((2 \pi+\gamma) / 2)=$ $-\cos (\gamma / 2)$.

The quaternion multiplication rule follows from Eqs. (3) and (4). For the product $\mu=\sigma \rho$, one obtains

$$
\begin{aligned}
& \mu_{1}=+\sigma_{1} \rho_{4}+\sigma_{2} \rho_{3}-\sigma_{3} \rho_{2}+\sigma_{4} \rho_{1}, \\
& \mu_{2}=-\sigma_{1} \rho_{3}+\sigma_{2} \rho_{4}+\sigma_{3} \rho_{1}+\sigma_{4} \rho_{2}, \\
& \mu_{3}=+\sigma_{1} \rho_{2}-\sigma_{2} \rho_{1}+\sigma_{3} \rho_{4}+\sigma_{4} \rho_{3}, \\
& \mu_{4}=-\sigma_{1} \rho_{1}-\sigma_{2} \rho_{2}-\sigma_{3} \rho_{3}+\sigma_{4} \rho_{4} .
\end{aligned}
$$

Finite rotations do not commute and this, of course, is reflected by the quaternion multiplication rules: $\sigma \rho \neq \rho \sigma$. The back rotation is expressed by $\tau^{-1}=\left(-\tau_{1},-\tau_{2},-\tau_{3},+\tau_{4}\right)$ with $\tau^{-1} \tau=\tau \tau^{-1}=\tau_{0}$. The quaternion $\tau_{0}=(0,0,0,1)$ denotes the identity transformation, i.e., no rotation at all. Let us, by convention, agree that the tetrahedron is in its standard orientation, which is shown in Fig. 1, if $\tau=\tau_{0}$.

\section{THE KINETIC ENERGY OF FULLY PROTONATED, DEUTERATED, AND PARTIALLY DEUTERATED METHANES}

For the description of the rotations of a rigid body (here of a molecule) in terms of quaternions, we use three coordinate systems (see Fig. 1). One is fixed in space, it is denoted by capital letters $(X, Y, Z)$. The second one is fixed in the molecule and rotates with it. The coordinate axes of the latter are denoted by the lower case letters $(x, y, z)$. In this frame, the tetrahedron is in its standard orientation. The third system 
also rotates with the molecule, its axes $(\mathbf{a}, \mathbf{b}, \mathbf{c})$ coincide with the principal axes of the moment of inertia $\boldsymbol{\Theta}$ $=\left(\Theta_{a}, \Theta_{b}, \Theta_{c}\right)$. Relative to the coordinate system $(x, y, z)$, the direction cosines of $(\mathbf{a}, \mathbf{b}, \mathbf{c})$ can be chosen as

$$
\begin{aligned}
& \mathbf{a}=(+1,-1,0) / \sqrt{2}, \\
& \mathbf{b}=(+1,+1,-2) / \sqrt{6}, \\
& \mathbf{c}=(+1,+1,+1) / \sqrt{3}
\end{aligned}
$$

for both $\mathrm{CH}_{3} \mathrm{D}$ and $\mathrm{CHD}_{3}$, with $\boldsymbol{\Theta}=(11,11,8) m a^{2} / 3$ and $\boldsymbol{\Theta}=(13,13,16) m a^{2} / 3$, respectively. Here, $m$ is the proton mass and $a$ the $\mathrm{C}-\mathrm{H}$ distance.

For $\mathrm{CH}_{2} \mathrm{D}_{2}$, the direction cosines are

$$
\begin{aligned}
& \mathbf{a}=(+1,+1,0) / \sqrt{2}, \\
& \mathbf{b}=(-1,+1,0) / \sqrt{2}, \\
& \mathbf{c}=(0,0,+1),
\end{aligned}
$$

with $\Theta=(12,10,14) m a^{2} / 3$.

For the spherical rotors $\mathrm{CH}_{4}$ and $\mathrm{CD}_{4}$, the axes $(\mathbf{a}, \mathbf{b}, \mathbf{c})$ may be chosen parallel to $(x, y, z)$ with the three components $\Theta_{a}=\Theta_{b}=\Theta_{c}==8 m a^{2} / 3$ and $16 m a^{2} / 3$, respectively.

For the calculation of the potential energy, the relative orientation of the standard molecular frame $(x, y, z)$ with respect to the space fixed coordinate system $(X, Y, Z)$ will be denoted by the quaternion $\tau$. For the calculation of the kinetic energy, we use the molecular frame $(\mathbf{a}, \mathbf{b}, \mathbf{c})$. Its orientation with respect to $(X, Y, Z)$ at time $t$ is expressed by the quaternion $\sigma=\left(\sigma_{1}, \sigma_{2}, \sigma_{3}, \sigma_{4}\right)$. At the instant of time $t$, the molecule rotates with an angular velocity $\omega=\left(\omega_{a}, \omega_{b}, \omega_{c}\right)$ around its principal axes $(\mathbf{a}, \mathbf{b}, \mathbf{c})$. Therefore, an infinitesimal time span $d t$ later, the molecule is found in the orientation

$$
\sigma+\dot{\sigma} d t=\rho \sigma
$$

where $\rho=\left(\dot{\rho}_{1} d t, \dot{\rho}_{2} d t, \dot{\rho}_{3} d t, 1-\epsilon\right)=\left(\omega_{a} d t / 2, \omega_{b} d t / 2, \omega_{c} d t / 2,1\right.$ $-\epsilon)$ is the quaternion for three joint infinitesimal rotations around the principal axes [see Eq. (6)]. $\quad \epsilon=\left(\omega_{a}^{2}+\omega_{b}^{2}\right.$ $\left.+\omega_{c}^{2}\right) d t^{2} / 4$ is of order $d t^{2}$ and will be dropped.

We solve Eq. (10) for $\rho$ with the result

$$
\rho=(\sigma+\dot{\sigma} d t) \sigma^{-1}=\tau_{0}+\dot{\sigma} \sigma^{-1} d t,
$$

where $\sigma^{-1}=\left(-\sigma_{1},-\sigma_{2},-\sigma_{3}, \sigma_{4}\right)$ and $\tau_{0}=(0,0,0,1)$ is the unit element of quaternion multiplication.

From the second term in Eq. (11), which is first order in $d t$, and from the rules of quaternion multiplication, one obtains the four equations $\dot{\rho}=\dot{\sigma} \sigma^{-1}$,

$$
\begin{aligned}
& \dot{\rho}_{1}=\omega_{a} / 2=+\dot{\sigma}_{1} \sigma_{4}-\dot{\sigma}_{2} \sigma_{3}+\dot{\sigma}_{3} \sigma_{2}-\dot{\sigma}_{4} \sigma_{1}, \\
& \dot{\rho}_{2}=\omega_{b} / 2=+\dot{\sigma}_{1} \sigma_{3}+\dot{\sigma}_{2} \sigma_{4}-\dot{\sigma}_{3} \sigma_{1}-\dot{\sigma}_{4} \sigma_{2}, \\
& \dot{\rho}_{3}=\omega_{c} / 2=-\dot{\sigma}_{1} \sigma_{2}+\dot{\sigma}_{2} \sigma_{1}+\dot{\sigma}_{3} \sigma_{4}-\dot{\sigma}_{4} \sigma_{3}, \\
& \dot{\rho}_{4}=0=+\dot{\sigma}_{1} \sigma_{1}+\dot{\sigma}_{2} \sigma_{2}+\dot{\sigma}_{3} \sigma_{3}+\dot{\sigma}_{4} \sigma_{4} .
\end{aligned}
$$

The last one of these equations is merely an expression of the fact that $\sigma$ is restricted to the surface of the four- dimensional unit sphere, i.e., the velocity is orthogonal on the radius vector and the four-dimensional (4D) scalar product of $\dot{\sigma}$ and $\sigma$ must vanish.

The Lagrangian form of the kinetic energy is

$$
K=\frac{1}{2} \sum_{i=1}^{3} \Theta_{i} \omega_{i}^{2}=2 \sum_{i=1}^{3} \Theta_{i} \dot{\rho}_{i}^{2} .
$$

The first three of Eq. (12) are inserted into Eq. (13) for the angular velocities in order to obtain the momenta conjugate to $\sigma_{\mu}$,

$$
p_{\mu}=\sum_{i=1}^{3} \frac{\partial K}{\partial \dot{\rho}_{i}} \frac{\partial \dot{\rho}_{i}}{\partial \dot{\sigma}_{\mu}} .
$$

This is evaluated with the help of Eq. (12),

$$
\begin{aligned}
& p_{1}=4\left(+\Theta_{1} \dot{\rho}_{1} \sigma_{4}+\Theta_{2} \dot{\rho}_{2} \sigma_{3}-\Theta_{3} \dot{\rho}_{3} \sigma_{2}\right), \\
& p_{2}=4\left(-\Theta_{1} \dot{\rho}_{1} \sigma_{3}+\Theta_{2} \dot{\rho}_{2} \sigma_{4}+\Theta_{3} \dot{\rho}_{3} \sigma_{1}\right), \\
& p_{3}=4\left(+\Theta_{1} \dot{\rho}_{1} \sigma_{2}-\Theta_{2} \dot{\rho}_{2} \sigma_{1}+\Theta_{3} \dot{\rho}_{3} \sigma_{4}\right), \\
& p_{4}=4\left(-\Theta_{1} \dot{\rho}_{1} \sigma_{1}-\Theta_{2} \dot{\rho}_{2} \sigma_{2}-\Theta_{3} \dot{\rho}_{3} \sigma_{3}\right) .
\end{aligned}
$$

Equation (15) is solved for the velocities $\dot{\rho}_{i}$ by the following linear combinations:

$$
\begin{aligned}
& P_{1}:=+p_{1} \sigma_{4}-p_{2} \sigma_{3}+p_{3} \sigma_{2}-p_{4} \sigma_{1}=4 \Theta_{1} \dot{\rho}_{1}, \\
& P_{2}:=+p_{1} \sigma_{3}+p_{2} \sigma_{4}-p_{3} \sigma_{1}-p_{4} \sigma_{2}=4 \Theta_{2} \dot{\rho}_{2}, \\
& P_{3}:=-p_{1} \sigma_{2}+p_{2} \sigma_{1}+p_{3} \sigma_{4}-p_{4} \sigma_{3}=4 \Theta_{3} \dot{\rho}_{3} .
\end{aligned}
$$

The velocities $\dot{\rho}_{i}$ from the right-hand side (rhs) of Eq. (16) are inserted into $K$ from Eq. (13) with the result

$$
\mathcal{K}=\frac{1}{8} \sum_{i=1}^{3} \frac{P_{i}^{2}}{\Theta_{i}} .
$$

This is the Hamiltonian form for the kinetic energy of a general asymmetric top ${ }^{12}$ in terms of the coordinates $\sigma_{\mu}$ and the corresponding conjugate momenta $p_{\mu}$ [Eq. (14)]. In the $\tau$ representation, the momentum operators $p_{\mu}$ assume the form $p_{\mu}=-i \hbar \partial / \partial \sigma_{\mu}$.

For a spherical top, $\mathcal{K}$ can be simplified to the wellknown expression

$$
\mathcal{K}=\frac{1}{8 \Theta}\left\{\sum_{\mu=1}^{4} p_{\mu}^{2}-3 \frac{\hbar}{i} \sum_{\mu=1}^{4} \tau_{\mu} p_{\mu}-\sum_{\mu, \nu=1}^{4} \tau_{\mu} \tau_{\nu} p_{\mu} p_{\nu}\right\} .
$$

\section{POCKET STATES}

\section{A. Expedient pocket states for a one-dimensional problem}

In this chapter, we come back to the pocket states of Ref. 16 which are restricted to a single pocket. They will be motivated with different arguments. Starting point for the calculation of the tunneling frequencies is the tight binding approximation of solid state physics. Its application to 
rotational tunneling will be explained for the example of the rotation in a one-dimensional periodic potential with the property

$$
V(x+2 \pi / 3)=V(x) .
$$

$V(x)=-A_{3} \cos (3 x)$ would be a typical potential for a rotating methyl group. There, $x$ is the rotation angle. The stationary Schrödinger equation for a particle of mass $m$ in the potential $V(x)$,

$$
-\frac{\hbar^{2}}{2 m} \frac{d^{2}}{d x^{2}} \psi(x)+V(x) \psi(x)=E \psi(x),
$$

is solved for periodic boundary conditions

$$
\psi(x+N \cdot 2 \pi / 3)=\psi(x)
$$

where $N$ is an integer. If $x$ is a rotation angle and $m$ the moment of inertia $\Theta$, and if $N=3$, then Eq. (20) is the stationary Schrödinger equation for the rotation of a methyl group in its threefold potential. In this case, the ground state multiplet consists of three states: An $A$ state and a doubly degenerate $E$ state. They represent the lowest lying band. In the tight binding (tunneling) limit, the corresponding energies are $E_{A}=E_{0}+2 h$ and $E_{E}=E_{0}-h$, where $h \leqslant 0$ is the tunneling matrix element. $E_{0}$ is an unimportant constant which depends on the choice of the zero point on the energy scale. $E_{E}-E_{A}=-3 h$ is the tunnel splitting.

For all values of $N$ in Eq. (21), the ground state is at the energy $E_{-}=E_{A}=E_{0}+2 h$. It is the lowest state in the band with $N$ members. The corresponding wave function $\psi_{-}(x)$ has positive (and equal) amplitude in every pocket. Its slope $d \psi_{-}(x) / d x$ vanishes at the barriers. This may also be called the bonding boundary condition. In the case of the model potential $V(x)=-A_{3} \cos (3 x)$ with $A_{3} \geqslant 0$, the barriers are found at the positions $x=x_{\nu}=(2 \nu+1) \cdot \pi / 3$, where $\nu$ is an integer.

In order to determine the overlap matrix element $h$, we need a state with antibonding boundary conditions at all barriers. Such a state does not exist for methyl rotations where the potential has a threefold symmetry. Therefore, we take refuge to an artificial problem with an even number of potential pockets.

For all even values of $N$, the maximum energy in the lowest lying band occurs for the state $\psi_{+}$with the antibonding boundary condition. In $\psi_{+}(x)$, the sign of the amplitude alternates from pocket to pocket and consequently $\psi_{+}(x)$ has nodes at $x=x_{\nu}$, i.e., at the positions of the barriers. The corresponding energy is $E_{+}=E_{0}-2 h$.

Using the Ritz variational principle, approximate values for the energies $E_{-}$and $E_{+}$can be calculated. This is highly efficient as the test functions have to be defined only in the range of $a$ single pocket, ${ }^{16}$ i.e., between the barriers at $x=$ $-\pi / 3$ and $x=+\pi / 3$. The boundary condition $d \psi_{-}(x) / d x=0$ at $x= \pm \pi / 3$ has to be fulfilled for the calculation of $E_{-}$, whereas $\psi_{+}( \pm \pi / 3)=0$ has to be enforced for the determination of $E_{+}$.

Once $E_{-}$and $E_{+}$have been found, one obtains the overlap matrix element $h$ from

$$
h=\left(E_{-}-E_{+}\right) / 4 \text {. }
$$

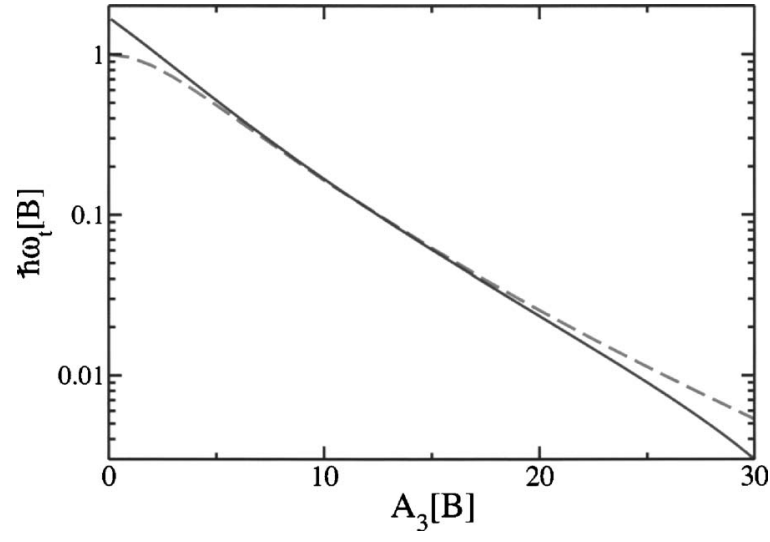

FIG. 2. The logarithm of the tunneling splitting $E_{E}-E_{A}=\hbar \omega_{t}=-3 h$ of a methyl group vs the potential strength $A_{3}$. A comparison of the pocket state approximation with the numerically exact solution of Mathieu's equation (dashed lines). Both $A_{3}$ and $\hbar \omega_{t}$ are measured in units of the rotational constant $B$.

In order to demonstrate the usefulness of the method, we calculate the tunnel splitting of a methyl group in the potential $V(x)=-A_{3} \cos (3 x)$, where $A_{3}$ is measured in units of the rotational constant $B=\hbar^{2} / 2 \Theta$. As test wave functions for the two different boundary conditions, we use

$$
\psi_{ \pm}(x)=\exp \left(-\frac{1}{2} \frac{x^{2}}{\Gamma_{ \pm}^{2}}\right)+C_{2}^{ \pm} x^{2}+C_{4}^{ \pm} x^{4} .
$$

As $\psi_{ \pm}(-x)=\psi_{ \pm}(x)$, the boundary condition needs to be applied only at one of the two saddles.

The Gaussian in Eq. (23) enables the optimal adaptation of the wave function in the minimum of the potential, whereas the following two terms allow its adjustment in the vicinity of the barrier. $C_{4}^{+}$is chosen such that $\psi_{+}(\pi / 3)=0$ is fulfilled and $C_{4}^{-}$is chosen such that $d \psi_{-}(x) / d x=0$ is fulfilled at $x=\pi / 3$. The remaining two constants, $\Gamma_{ \pm}$and $C_{2}^{ \pm}$, for the respective wave functions are the variational parameters in the minimalization of

$$
E_{ \pm}=\frac{\left\langle\psi_{ \pm}|H| \psi_{ \pm}\right\rangle}{\left\langle\psi_{ \pm} \mid \psi_{ \pm}\right\rangle},
$$

where $H=-\left(\hbar^{2} / 2 m\right)\left(d^{2} / d x^{2}\right)+V(x)$ is the Hamiltonian of Eq. (20) and where the scalar products are obtained as integrals over $-\pi / 3<x \leqslant \pi / 3$.

Figure 2 shows a comparison of $\hbar \omega_{t}=\log (-3 h)$ from Eq. (22) with the logarithm of the numerically exact tunneling frequency $E_{E}-E_{A}$, as calculated from Mathieu's equation [Eq. (20)]. There is sufficiently good agreement for $5<A_{3}$ $<20$. It is perfectly understandable that there are strong deviations for $A_{3}<5$ outside the tunneling (or tight binding) regime. It would be an easy matter to extend the range of validity for larger $A_{3}$, beyond $A_{3}=20$, by including a few more power terms into expression (23) for the wave function. It is, however, not our intention to find another nearly exact solution of Mathieu's equation in one dimension but to demonstrate the merits and limitations of these pocket states. ${ }^{16}$ In the following, the method will be applied to the problem of the three-dimensional rotation of molecules where no exact solutions are available. 


\section{B. Expedient pocket states for methane rotations}

In phase III of methane which will be described below (Sec. VI), there are two sites of different symmetries: The $m$ site and the 2 site. Even permutations of the four corners (protons or deuterons) of the $\mathrm{CH}_{4}$ or $\mathrm{CD}_{4}$ tetrahedron lead to equivalent orientations, e.g., into equivalent minima, and this is independent of the symmetry of the site. There are 12 even permutations of 4 objects and thus there are 12 equivalent minima or pockets of the potential. As the quaternions cover the space of orientations twice (antipodal points on the 4D unit sphere belong to the same orientation), there are 24 pockets in quaternion space. When the tetrahedron (together with its surrounding) is rotated such that one of the minima corresponds to its standard orientation $\tau_{0}$, then 8 of these pockets are found in the positions $\tau$ $=( \pm 1,0,0,0),(0, \pm 1,0,0),,(0,0, \pm 1,0),(0,0,0, \pm 1), \quad$ and the other 16 in the positions $\tau=( \pm z, \pm z, \pm z, \pm z)$ with $z=\frac{1}{2}$. Starting in the pocket of the standard orientation, rotations by $\pm 120^{\circ}$ around the four threefold symmetry axes across saddle points at $\tau_{s}=( \pm t, \pm t, \pm t,+r)$ with $t=(1 / 12)^{1 / 2}$ and $r$ $=(3 / 4)^{1 / 2}$ lead into the eight closest pockets at $( \pm z, \pm z, \pm z$, $+z$ ). Thus, the 24 pockets form a finite three-dimensional bcc lattice which is embedded into a curved three-dimensional (3D) space, the surface of the 4D unit sphere.

The variational wave functions $\psi_{a b c d}(\tau)$ (with $a, b, c, d$ $=+,-$ ) shall be exploited only on a spherical (pseudo)cap on the surface of the $4 \mathrm{D}$ unit sphere. The cap is a 3D sphere, it is centered at $\tau_{0}$, and reaches out to the saddle points, i.e., it is limited by $\tau_{1}^{2}+\tau_{2}^{2}+\tau_{3}^{2} \leqslant \frac{1}{4}$ (or equivalently by $1 \geqslant \tau_{4} \geqslant \sqrt{\frac{3}{4}}$ ). The volume of the cap is $V_{c}=\pi^{2} / 3-\sqrt{\frac{3}{4}} \pi \simeq 0.569169$ (see the Appendix). The 24 caps centered at the 24 minima of the potential and touching each other at the saddle points would fill $69 \%$ of the whole quaternion space of volume $V_{0}=2 \pi^{2}$. This comes rather close to the $68 \%$ filling factor of the infinite bcc lattice in a flat geometry. One great advantage over other pocket state approaches, where the test wave function had to be optimized in the whole quaternion space, is that now the procedure must no longer be applied to the full space but only to one of the 24 caps, i.e., to only $3 \%$ of it.

The quaternions at four of the eight saddle points are denoted by

$$
\begin{aligned}
& \tau_{a}=(+t,-t,-t,+r), \\
& \tau_{b}=\left(\begin{array}{ll}
-t,+t,-t,+r
\end{array}\right), \\
& \tau_{c}=\left(\begin{array}{ll}
-t,-t,+t,+r
\end{array}\right), \\
& \tau_{d}=(+t,+t,+t,+r) .
\end{aligned}
$$

The values of the wave functions at the other four saddles are related to these by symmetry [see the remark immediately below Eq. (23)].

The indices $a, b, c, d=+,-$ in $\psi_{a b c d}(\tau)$ differentiate between the bonding "-" and antibonding "+" boundary conditions at the respective saddle points. If, e.g., the index $a$ is + , then $\psi_{a b c d}\left(\tau_{a}\right) \rightarrow \psi_{+b c d}\left(\tau_{a}\right)=0$.

If $a$ is - , then the directional derivative

$$
\psi_{a b c d}^{d}(\tau)=\sum_{i=1}^{4} \tau_{i} \frac{\partial \psi_{a b c d}(\tau)}{\partial \tau_{i}}
$$

of $\psi_{a b c d}(\tau)$ along the direction from $\tau_{0}$ to $\tau$ is zero at $\tau=\tau_{a}$ : $\psi_{-b c d}^{d}\left(\tau_{a}\right)=0 . \psi_{----}(\tau)$ is the ground state with energy $E_{---}$ and

$$
E_{+b c d}-E_{-b c d}=-4 h_{a}
$$

permits the calculation of the tunneling matrix element $h_{a}$ for rotations around the $(+1,-1,-1)$ axis and, respectively, for the indices $b, c$, and $d$. The maximum splitting between the highest level $(E)$ and the lowest level $(A)$ level totals $-3\left(h_{a}\right.$ $\left.+h_{b}+h_{c}+h_{d}\right)$.

\section{THE VARIATIONAL WAVE FUNCTIONS}

The variational wave functions $\psi_{a b c d}(\tau)$ which are used in the following for the determination of the tunnel splitting in the ground state multiplet do not depend explicitly on $\tau_{4}$ but only on $\tau_{1}, \tau_{2}$, and $\tau_{3}$. They depend only implicitly on $\tau_{4}$ as the four components of $\tau$ are interconnected via their normalization condition [Eq. (5)].

In order to render the ansatz for $\psi_{a b c d}(\tau)$ as flexible as possible in the directions toward the saddle points, where the potential is softest and where the most important overlap occurs, we introduce a new four-component variable $T$ $=\left(T_{1}, T_{2}, T_{3}, T_{4}\right)$ by

$$
\begin{aligned}
& T_{1}=+\tau_{1}-\tau_{2}-\tau_{3}, \\
& T_{2}=-\tau_{1}+\tau_{2}-\tau_{3}, \\
& T_{3}=-\tau_{1}-\tau_{2}+\tau_{3}, \\
& T_{4}=+\tau_{1}+\tau_{2}+\tau_{3} .
\end{aligned}
$$

Any one of the wave functions $\psi_{a b c d}(\tau)$ is expressed in terms of $T: \psi(\tau)=\Phi[T(\tau)]$, where

$$
\Phi[T]=\exp \left(-\frac{1}{2} \sum_{i=1}^{4} \frac{T_{i}^{2}}{\Gamma_{i}^{2}}\right)+\sum_{i=1}^{4}\left[A_{i} T_{i}^{K}+B_{i} T_{i}^{L}\right] .
$$

Typical values of $K$ and $L>K$ are 4, 6, 8, etc. In order to avoid some of the clumsiness of the notation, we have omitted the indices abcd for the moment. These will be reintroduced when the boundary conditions are discussed.

Inserting the saddle point coordinates $\tau_{a}, \tau_{b}, \tau_{c}, \tau_{d}$ from Eq. (25) into the rhs of Eq. (28), one obtains

$$
\begin{aligned}
& T_{a}=(3 t,-t,-t,-t), \\
& T_{b}=(-t, 3 t,-t,-t), \\
& T_{c}=(-t,-t, 3 t,-t), \\
& T_{d}=(-t,-t,-t, 3 t) .
\end{aligned}
$$

At the saddle point at $T_{a}$, the value of $T_{\mathrm{a} 1}^{K}$ in Eq. (29) by far exceeds the other three terms: $T_{a 1}^{K} \gg T_{a 2}^{K}, T_{a 3}^{K}, T_{a 4}^{K}$ when $K=4$ or larger. Therefore, a large value of $A_{1}$ in Eq. (29) favors a pronounced lobe of the wave function in the vicinity 
of the saddle point at $T_{a}$, i.e., a high amplitude for angular excursions of the molecule around the $(+1,-1,-1)$-rotation axis. In the same way, the coefficients $A_{2}, A_{3}, A_{4}$ are responsible for the amplitude of $\psi$ for rotations around the other threefold rotation axes of the molecule.

A little bit of algebra shows that

$$
\sum_{i=1}^{4} \tau_{i} \frac{\partial \psi(\tau)}{\partial \tau_{i}}=\sum_{j=1}^{4} T_{j} \frac{\partial \Phi(T)}{\partial T_{j}}-\frac{1}{2}\left(\sum_{k=1}^{4} T_{k}\right)\left[\sum_{m=1}^{4} \frac{\partial \Phi(T)}{\partial T_{m}}\right]
$$

As $\sum_{k=1}^{4} T_{k}=0$ at the four saddle points, the boundary condition $\psi^{d}(\tau)=0$ can be replaced by $\Phi^{d}(T)=0$ for $x$ $=a, b, c, d$, i.e., at the positions of the four saddle points. $\psi^{d}\left(\tau_{x}\right)$ is the left-hand side of Eq. (31) and $\Phi^{d}\left(T_{x}\right)$ is defined by the first term on the rhs.

The four parameters $B_{i}$ are adjusted such that the four boundary conditions in the directions of the saddle points are fulfilled. The four $\Gamma_{i}$ and the four $A_{i}$ are the eight variational parameters in the search for the minimum expectation value of the energy.

\section{A MODEL POTENTIAL}

The structure of phase II of solid methane has been predicted by James and Keenan ${ }^{18}$ on the basis of the electrostatic octopole-octopole interaction of the molecules, using the Ornstein-Zernicke approximation. There, the fluctuations are considered to be independent of each other. The orientational fluctuations in the orientationally disordered phase I are the precursors of the structure which becomes stable when the temperature is lowered below $20.4 \mathrm{~K}$. At the transition temperature, they condense into the static distortion which amounts to the order parameter of the new phase II. The predicted structure, ${ }^{18,19}$ the geometrical nature of the fluctuations, ${ }^{20}$ and their critical slowing down ${ }^{21}$ have later all been verified experimentally.

All attempts to predict the structure of phase III of heavy methane by the same kind of interaction have failed, ${ }^{23,24}$ but in 2003, this structure has finally been determined experimentally. ${ }^{17}$ It is orthorhombic with space group $\mathrm{Cmca}$ (lattice parameters $a=11.7079 \AA, \quad b=8.1893 \AA$, and $c$ $=8.1842 \AA$ ), with eight molecules in the unit cell. Groups of four molecules are related by symmetry. While the phase III structure is not a subgroup of that of phase II $[F m 3 c$ (Refs. 18 and 19)], the center of mass structure still can be considered as a distorted fcc lattice. It is convenient to approximate the methane molecules by rigid tetrahedra: Of course, minor distortions at the sites of low symmetry are permitted. The carbon atoms in the molecular center are displaced with respect to a fcc lattice, with two independent vector components at the $m$ site and just one at the 2 site. This is probably the reason why there was no successful theoretical prediction $^{23,24}$ of the structure of phase III. In order to be successful, it seems obligatory to include these center of mass (c.m.) displacements.

The orientational order of phase III is more important for our consideration. With respect to the orthorhombic unit cell, fictitious high symmetry tetrahedral orientations can be taken as a reference. Then, the actual orientations can be described as deviations from these high symmetry orientations by the introduction of rotation angles. Only one free parameter (angle) for each of the two sites is needed; (i) the angle $\beta$ for rotation around the normal to the mirror plane (through the $\mathrm{C}$ atom) at the $m$ site and (ii) the angle $\alpha$ for rotation around the twofold axis at the other site.

All five parameters have been determined by neutron diffraction $^{17}$ but of course with a non-negligible statistical error.

Now that the structure has been determined, the question if there exists a model potential that is consistent with this structure and which at the same time explains the tunneling splitting $^{25}$ of the molecules at the two sites with different symmetries is self-imposing.

Let us start from a modified Coulomb potential for the atom-atom interaction,

$$
V=\frac{1}{2} \sum_{i, j} \frac{Q_{i} Q_{j}}{\left|\mathbf{R}_{i}-\mathbf{R}_{j}\right|^{n}}
$$

where $V$ is the orientational potential energy of the crystal. Here, $\mathbf{R}_{i}$ and $\mathbf{R}_{j}$ are the positions of the nuclei (in different molecules). If $n=1$ and if the charges are chosen as $Q_{i}=+1$ and $Q_{i}=-4$ for hydrogen and carbon, respectively, it would be the Coulomb case. It will turn out that higher values of $n$ are needed for a satisfactory agreement with experiment. This indicates that it is the short range repulsion of the electron shells which is responsible for the orientational order. For large values of $n$, only the shortest intermolecular $\mathrm{H}-\mathrm{H}$ distances are of importance, but we retain the form of Eq. (32) with the same "charges" in order to also include the Coulomb case.

The interaction in Eq. (32) lacks the attractive dispersion forces and hence it is by no means adequate for predicting the center of mass structure. The carbon positions are therefore taken from the experiment and then we look for the equilibrium orientations of both molecules.

After all these considerations of a more general nature, now comes the application, namely, the calculation of the molecular orientations in the experimentally determined c.m. lattice.

Using the interaction in Eq. (32), we calculate the (orientational) potential energy $V_{\xi}$ for one molecule of each kind,

$$
V_{\xi}(\alpha, \beta)=\sum_{i=1}^{5} Q_{i} \sum_{j=1}^{60} \frac{Q_{j}}{\left|\mathbf{R}_{i}-\mathbf{R}_{j}\right|^{n}},
$$

with $\xi=m$ or 2 , distinguishing the two sites of different symmetries. The first sum in Eq. (33) runs over the five atoms of the central molecule, and the second sum runs over the 60 atoms in the shell of its 12 nearest neighbor (nn) molecules.

The angles $\alpha$ and $\beta$ of the central molecule are varied in steps of $1^{\circ}$ while, at the same time, the 12 molecules at the $m$ and 2 sites in the nn shells are rotated correspondingly. We look for the minima of the energy per molecule, 


$$
S(\alpha, \beta)=\left(V_{m}(\alpha, \beta)+V_{2}(\alpha, \beta)\right) / 4,
$$

among the $360 \times 360$ values on the grid of angles $\alpha$ and $\beta$. There are quite a few local minima with rather similar $S(\alpha, \beta)$. For all values of $n \geqslant 1$, there is one minimum in the immediate vicinity of the experimentally determined values $\alpha_{x}=5.2^{\circ}$ and $\beta_{x}=40.6^{\circ}$. In the Coulomb case $(n=1)$, this is not the absolute minimum. Its position does not move a lot when $n$ is varied, but it becomes the absolute minimum for all values of $n \simeq 2.12$. This is a rather strong indication that the orientational order of phase III is a result of the short range repulsion or steric hindrance of the molecules.

A consecutive minimization procedure for $S(\alpha, \beta)$ improves the values $\alpha_{n}$ and $\beta_{n}$ on the grid. For $n=8$, one obtains $\alpha_{8}=9.5^{\circ}$ and $\beta_{8}=41.9^{\circ}$. Attempts to gain even better agreement with the experimental values $\alpha_{x}$ and $\beta_{x}$ by a variation of the c.m. displacements, within their error bars, did not show clear trends and have therefore been abandoned. When the angles $\alpha_{n}$ and $\beta_{n}$ have been determined, the orientational potential can be calculated for arbitrary orientations of the central molecule while its 12 nn's are kept fixed in their equilibrium orientation.

\section{THE GROUND STATE MULTIPLET}

Having collected all the ingredients, we can finally proceed to calculate the energy levels in the ground state multiplet. The Ritz variational principle shall be applied to minimize

$$
E_{a b c d}=\frac{\left\langle\psi_{a b c d}\left|\mathcal{H}_{\xi}\right| \psi_{a b c d}\right\rangle}{\left\langle\psi_{a b c d} \mid \psi_{a b c d}\right\rangle}
$$

The wave functions $\psi_{a b c d}$ have been introduced in Sec. V. The Hamiltonian for the two sites with $\xi=m$ and $\xi=2$ is

$$
\mathcal{H}_{\xi}(\tau, p)=\mathcal{K}(\tau, p)+s \mathcal{V}_{\xi}(\tau),
$$

where $\mathcal{K}(\tau, p)$ is the kinetic energy from Sec. III. In the Coulomb case $(n=1)$, an estimate for the strength parameter $s$ might be provided from effective charges $Q_{i}$ somewhat smaller than the bare charges (screening by the electron shells), but for the heuristic $1 / r^{n}$ potential, there is no chance for guessing its value: $s$ is an adjustable fitting parameter. For $\mathrm{CH}_{4}$ and $\mathrm{CD}_{4}$, the coordinate systems $(x, y, z)$ and $(a, b, c)$ coincide and $\tau=\sigma, \mathcal{K}(\tau, p)$ can be taken directly from Eq. (17), but for the partially deuterated molecules one has to substitute $\sigma$ by the appropriate linear combination $\sigma=\sigma(\tau)$ and the derivatives in the momenta $p_{i}[\mathrm{Eq}$. (16)] by

$$
\frac{\partial \psi}{\partial \sigma_{i}}=\sum_{j} \frac{\partial \psi}{\partial \tau_{j}} \frac{\partial \tau_{j}}{\partial \sigma_{i}}
$$

The strength parameter $s$ of the potential was not needed in Sec. VI where the determination of the equilibrium orientations $\alpha_{n}$ and $\beta_{n}$ was the only goal.

For the calculation of the potential energy $\mathcal{V}_{\xi}(\tau)$, we use the atom-atom interaction of Eq. (33) for a given $n$ and determine the angles $\alpha_{n}$ and $\beta_{n}$, as explained in Sec. VI. When the equilibrium has been found, we rotate the whole crystal rigidly into such an orientation that the molecule in the center is its standard orientation $\tau=\tau_{0}$. Then, its surroundings

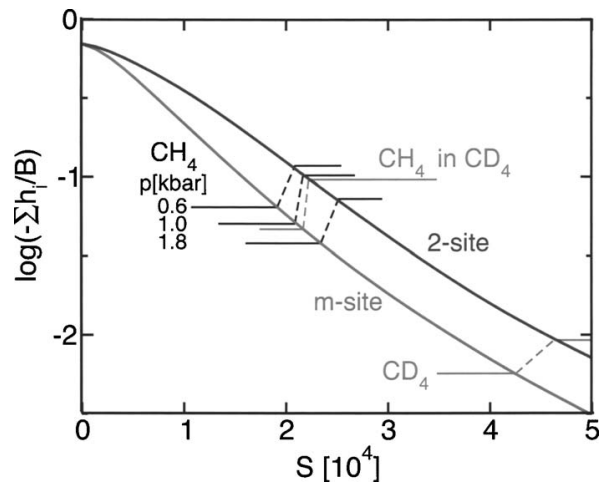

FIG. 3. The sum $\sum_{i} h_{i}$ of the four overlap matrix elements yields the total width of the tunneling multiplet $E_{E}-E_{A}=3 \Sigma_{i} h_{i}$. The figure shows the calculated values of this sum for both sites on a logarithmic scale as a function of the strength parameter $s$ of the potential. The horizontal lines show the experimentally determined values (Ref. 25) for different pressures and for $\mathrm{CD}_{4}$. Ideally, the strength parameter for the two symmetries should be the same, i.e., the broken lines should be vertical. As all energies are measured in units of $B$, the value of $s_{\mathrm{CD}_{4}}$ should be roughly $2 s_{\mathrm{CH}_{4}}$.

are kept fixed, while the potential energy $\mathcal{V}_{\xi}(\tau)$ of the central molecule is determined for $10^{6}$ equally distributed random values $\tau_{i}$ of $\tau$ on the $3 \%$ cap described in Sec. IV B, i.e., for $10^{6}$ random orientations within the potential pocket.

When the potential is plotted along the directions toward the saddle points [that is, for $\tau=( \pm \eta, \pm \eta, \pm \eta, \zeta)$ with $\eta$ $=\frac{1}{\sqrt{3}} \sin \gamma / 2$ and $\zeta=\cos \gamma / 2$, one realizes that its shape is almost independent of the exponent $n$. So, $n$ is not really an adjustable parameter. Of course, the strength parameter $s$ varies strongly with $n$. The same $s$ should apply for both symmetries.

The expectation value [Eq. (35)] of the energy is approximated by

$$
E_{a b c d}=\frac{\sum_{i}\left\langle\psi_{a b c d}\left(\tau_{i}\right)\left|\mathcal{H}_{\xi}\right| \psi_{a b c d}\left(\tau_{i}\right)\right\rangle}{\sum_{i}\left\langle\psi_{a b c d}\left(\tau_{i}\right) \mid \psi_{a b c d}\left(\tau_{i}\right)\right\rangle}
$$

where the sums over $i$ run over the $10^{6}$ random values of $\tau_{i}$. With $a, b, c, d= \pm 1$, there are 16 values of $E_{a b c d}$ for the different boundary conditions at the four saddle points. The eight variational parameters $\Gamma_{i}$ and $A_{i}$ are varied for each one of the $E_{a b c d}$ until their minima have been found. From their differences one obtains the tunneling matrix elements [Eq. (27)].

The first example for a particle in the potentials $V_{2}(\tau)$ or $V_{m}(\tau)$ is a regular tetrahedron, be it $\mathrm{CH}_{4}$ or $\mathrm{CD}_{4}$. As a start, we calculate

$$
\Sigma_{\xi}=\sum_{i=1}^{4} h_{i}=\left(E_{++++}-E_{----}\right) / 4 .
$$

In Fig. 3, the logarithms of the two sums $\Sigma_{2}$ and $\Sigma_{m}$, for either symmetry, are plotted as functions of the strength parameter $s$. Horizontal lines cut the graphs at the experimental values of $\log \left(\Sigma_{2}\right)$ and $\log \left(\Sigma_{m}\right)$ and yield the corresponding values of s. The five examples of Ref. 25 have been included. The corresponding strength parameters for the two symmetries do not differ by more than $8 \%$ from each other. This rather good agreement is better than anticipated. It must not be expected from such a rough model for the potential. The 
TABLE I. Tunneling matrix elements for $\mathrm{CH}_{4}(1.0 \mathrm{kbar})$ in $\mu \mathrm{eV}$.

\begin{tabular}{lcccccc}
\hline \hline & \multicolumn{2}{c}{2 site } & & \multicolumn{3}{c}{$m$ site } \\
\cline { 2 - 3 } \cline { 5 - 7 } Matrix element & $h_{1}=h_{3}$ & $h_{2}=h_{4}$ & & $h_{1}$ & $h_{2}$ & $h_{3}=h_{4}$ \\
\hline Expt. & -20 & -12 & & -2.7 & -16.5 & -7.4 \\
Model potential & -19 & -14 & & -0.6 & -23.8 & -2.9 \\
Surrogate potential & -20 & -12 & & -2.6 & -16.4 & -7.3 \\
\hline \hline
\end{tabular}

same is true for the comparison of the strength parameter for $\mathrm{CD}_{4}$ and for $\mathrm{CH}_{4}$ in $\mathrm{CD}_{4}$. In units of $B\left(\mathrm{CH}_{4}\right)=654 \mu \mathrm{eV}$ and $B\left(\mathrm{CD}_{4}\right)=327 \mu \mathrm{eV}$, the value of $s$ should differ by a factor of 2 for the two cases, which is almost perfectly fulfilled. This means that the potential strengths are equal in absolute units (e.g., $\mu \mathrm{eV}$ ).

Thus, the overall tunneling splitting of fully protonated and fully deuterated methane in phase III is rather well described in terms of the simple interaction model, but when it comes to the individual tunneling matrix elements, the agreement is less convincing.

In the Tables I and II (for $\mathrm{CH}_{4}$ and $\mathrm{CD}_{4}$ ) the row which is denoted by "model potential" shows that relatively large discrepancies occur for the smaller matrix elements. The latter do not contribute appreciably to the overall splitting and furthermore small variations of the barrier heights entail noticeable modifications of the overlap.

In order to acquire an idea how large the error in the potential barriers might be, we take refuge to a surrogate potential. This potential should be as simple as possible and it should depend on the minimum number of parameters. Observing the requirements of the respective site symmetry, the surrogate potential is constructed such that it yields the tunneling splitting for $\mathrm{CH}_{4}$ under a pressure of $1 \mathrm{kbar}$ with good precision. The result is denoted by "surrogate potential" and it is shown in Table I.

The surrogate potential is constructed as follows: There are four centers of repulsion at the positions $\mathbf{R}_{j}=(-a, a, a),(a,-a, a),(a, a,-a),(-a,-a,-a) \quad$ with $a$ $=2.8 \AA / \sqrt{3}$. Each one of these four centers of repulsion interacts with each one of the four protons of the methane molecule [see Eq. (40)]. In order to retain a little bit of similarity with the original, $\left|R_{j}\right|=2.8 \AA$ is chosen to be equal to the distance from the central carbon to the nearest protons of neighboring molecules in the real crystal. The interaction potential of the four centers with the four protons of the central methane molecule is taken to be

TABLE II. Tunneling matrix elements for $\mathrm{CD}_{4}$ in $\mu \mathrm{eV}$.

\begin{tabular}{lllllll}
\hline \hline & \multicolumn{2}{c}{2 site } & & \multicolumn{3}{c}{$m$ site } \\
\cline { 2 - 3 } \cline { 5 - 7 } Matrix element & $h_{1}=h_{3}$ & $h_{2}=h_{4}$ & & $h_{1}$ & $h_{2}$ & $h_{3}=h_{4}$ \\
\hline Expt. & -1.116 & -0.524 & -0.167 & -0.733 & -0.532 \\
Model potential & -1.06 & -0.82 & & -0.01 & -1.52 & -0.08 \\
Surrogate potential & -1.63 & -0.78 & -0.10 & -1.05 & -0.40 \\
\hline \hline
\end{tabular}

TABLE III. The barrier heights for both potentials in $\mu \mathrm{eV}$.

\begin{tabular}{lcccccc}
\hline \hline & \multicolumn{2}{c}{2 site } & & \multicolumn{3}{c}{$m$ site } \\
\cline { 2 - 3 } \cline { 5 - 7 } Barrier height & $B_{1}=B_{3}$ & $B_{2}=B_{4}$ & & $B_{1}$ & $B_{2}$ & $B_{3}=B_{4}$ \\
\hline Model potential & 19500 & 17800 & & 51400 & 32200 & 15300 \\
Surrogate potential & 16900 & 14400 & & 27500 & 21600 & 17000 \\
\hline \hline
\end{tabular}

$$
V=\sum_{i, j=1}^{4} A_{j}\left(\mathbf{R}_{j}-\mathbf{r}_{i}\right)^{-8},
$$

where the $\mathbf{r}_{i}$ are the positions of the protons of the methane molecule at the origin and the dimension of $\mathbf{A}_{j}$ is $\mu \mathrm{eV} \AA^{8}$.

In the case of the 2 site, the strength parameters $A_{j}$ are pairwise equal: $A_{1}=A_{3}$ and $A_{2}=A_{4}$. For the $m$ site, only $A_{1}$ $=A_{2}$ applies. For any choice of the $A_{j} \geqslant 0$, the minimum of the potential is at $\tau_{0}$ the normal orientation of the tetrahedron. The choice $A_{j}=(5159,3172,5159,3172)$ for the 2 site and $A_{j}=(6101,6101,1394,9761)$ for the $m$ site leads to the barrier heights shown in Table III.

The most pronounced error (by a factor of 2) occurs for $B_{1}$, the highest rotational barrier at the $m$ site, where the overlap is smallest. Nevertheless, we consider the discrepancies between the calculations and the experiments acceptable. First, the uncertainty in the experimental value of such small overlap matrix elements certainly is rather large. Second, the carbon positions have been taken from the experiment. Small uncertainties of these might have an appreciable effect on the barriers. Finally, as has been repeated several times, we do not expect a better agreement for our crude model.

Most of the observed crystallographic and of the tunneling features are well represented by the model.

\section{CONCLUSIONS}

Despite of the fact that $\mathrm{CH}_{4}$ is the simplest one of all organic molecules, solid methane exhibits a rich phase diagram. The structural modifications differ mainly in the orientational order of the methane tetrahedra. The pattern of ordering depends on the temperature, on the external pressure, and on the isotopic composition. Above $20.4 \mathrm{~K}(27 \mathrm{~K}$ for $\mathrm{CD}_{4}$ ), the $\mathrm{CH}_{4}$ crystal is orientationally disordered (phase I). Below, as a consequence of the interplay between the orientational forces and the thermal and quantum fluctuations, it is partially ordered (phase II). The center of mass structure in both phases is fcc as would be expected for solids composed of globular molecules. At even lower temperatures, $\mathrm{CD}_{4}$ (and $\mathrm{CH}_{4}$ under pressure) undergo a further transition into the fully ordered phase III whose structure was recently determined. There, the molecules occupy sites of two different symmetries ( $m$ site and 2 site). The center of mass structure departs from the ideal fcc structure but the displacements of the carbon atoms are small; they amount to a few percent of the lattice spacing.

The aim of the present paper was the verification of the orientational structure of phase III by an explanation of the rotational tunneling of the methane molecules at the two sites and that for both $\mathrm{CH}_{4}$ and $\mathrm{CD}_{4}$. Given the center of mass 
structure, short range repulsive forces between the hydrogen atoms can indeed succeed with both goals. Considering the fact that the tunneling frequencies depend exponentially, i.e., very sensitively, on the moment of inertia and the potential strength, this is rather reassuring.

It would be highly desirable to find a (quantum chemical) potential which also explains the center of mass structure. Such a potential must include the attractive forces between the molecules. This goes far beyond our intentions.

In the course of these calculations, we have extended the pocket state method to include also the tunneling of the partially deuterated methanes. The corresponding matrix elements are needed for the explanation of the existing data for $\mathrm{CHD}_{3}$ and they can be used for predicting the tunneling splitting of $\mathrm{CH}_{3} \mathrm{D}$ and of $\mathrm{CH}_{2} \mathrm{D}_{2}$. A complication arises from the translation-rotation coupling, i.e., from the fact that in a rotation of the $X_{2} Y$ triangle, the carbon also moves. These questions are reserved to a forthcoming paper on the partially deuterated methanes.

\section{APPENDIX: VOLUME OF THE CAP}

In order to determine the volume of the cap (of the pocket) on the surface of the four-dimensional unit sphere, a change to convenient variables is performed: The 4D Cartesian coordinates $\tau=\left(\tau_{1}, \tau_{2}, \tau_{3}, \tau_{4}\right)$ are replaced by the 4D polar coordinates $(r, \phi)=\left(r, \phi_{1}, \phi_{2}, \phi_{3}\right)$,

$$
\begin{aligned}
& \tau_{1}=r \sin \phi_{1} \sin \phi_{2} \sin \phi_{3}, \\
& \tau_{2}=r \sin \phi_{1} \sin \phi_{2} \cos \phi_{3}, \\
& \tau_{3}=r \sin \phi_{1} \cos \phi_{2}, \\
& \tau_{4}=r \cos \phi_{1},
\end{aligned}
$$

with $0 \leqslant \phi_{1} \leqslant \pi, 0 \leqslant \phi_{2} \leqslant \pi$, and $0 \leqslant \phi_{3} \leqslant 2 \pi$. An integral $\mathcal{J}_{4}$ over a $4 \mathrm{D}$ region $R$ in $\tau$ space is calculated as

$$
\begin{aligned}
\mathcal{J}_{4}= & \int_{R} F(\tau) d \tau_{1} d \tau_{2} d \tau_{3} d \tau_{4}=\int_{R} F(\tau(r, \phi)) \\
& \times\left|\frac{\partial\left(\tau_{1}, \tau_{2}, \tau_{3}, \tau_{4}\right)}{\partial\left(r, \phi_{2}, \phi_{2}, \phi_{3}\right)}\right| d r d \phi_{1} d \phi_{2} d \phi_{3},
\end{aligned}
$$

where

$$
\frac{\partial\left(\tau_{1}, \tau_{2}, \tau_{3}, \tau_{4}\right)}{\partial\left(r, \phi_{1}, \phi_{2}, \phi_{3}\right)}=r^{3} \sin ^{2} \phi_{1} \sin \phi_{2}
$$

is the Jacobian of the transformation.

If the integration is restricted to a $3 \mathrm{D}$ volume $S$ on the surface of the 4D unit sphere, one inserts the Dirac $\delta$ function $\delta(r-1)$ on the rhs of Eq. (A2), performs the $r$ integration, and obtains the integral $\mathcal{J}_{3}$,

$$
\mathcal{J}_{3}=\int_{S} F(\tau(1, \phi)) \sin ^{2} \phi_{1} \sin \phi_{2} d \phi_{1} d \phi_{2} d \phi_{3} .
$$

For the determination of the volume $\mathcal{V}_{\text {cap }}$ of a cap of variable size $q$ with $1 \geqslant \tau_{4} \geqslant q$, the function $F$ is set equal to 1 , identically, and the integration over $\phi_{1}$ is restricted to 0 $\leqslant \phi_{1} \leqslant \Lambda$ with $\Lambda=\arccos q$,

$$
\begin{aligned}
\mathcal{V}_{\text {cap }} & =\int_{0}^{\Lambda} \sin ^{2} \phi_{1} d \phi_{1} \int_{0}^{\pi} \sin \phi_{2} d \phi_{2} \int_{0}^{2 \pi} d \phi_{3}, \\
& =2 \pi\left(\Lambda-\frac{1}{2} \sin (2 \Lambda)\right) .
\end{aligned}
$$

The volume $V_{c}=\pi^{2} / 3-\pi \sqrt{\frac{3}{4}}$ of the pocket is obtained for $q$ $=\sqrt{\frac{3}{4}}$, i.e., for $\Lambda=\pi / 6$. The volume $V_{0}=2 \pi^{2}$ of the complete surface of the 4D unit sphere is obtained for $q=-1$, i.e., when $\Lambda=\pi$ is inserted into Eq. (A6).

${ }^{1}$ H. Wipf, A. Magerl, S. M. Shapiro, S. K. Satija, and W. Thomlinson, Phys. Rev. Lett. 46, 947 (1981).

${ }^{2}$ K. Neumaier, D. Steinbinder, H. Wipf, H. Blank, and G. Kearley, Z. Phys. B: Condens. Matter 76, 359 (1989).

${ }^{3}$ W. Press, Single-Particle Rotations in Molecular Crystals (Springer, Berlin, 1981), Vol. 92.

${ }^{4}$ M. Prager and A. Heidemann, Chem. Rev. (Washington, D.C.) 97, 2933 (1997).

${ }^{5}$ Springer Proceedings in Physics, edited by A. Heidemann (Springer, Berlin, 1987), Vol. 17.

${ }^{6}$ I. F. Silvera, Rev. Mod. Phys. 52, 393 (1980).

${ }^{7}$ W. F. Hardy and I. F. Silvera, Phys. Rev. Lett. 26, 127 (1971).

${ }^{8}$ W. R. Hamilton, Lectures on Quaternions: Containing a Systematic Statement of a New Mathematical Method (Hodges and Smith, Dublin, 1835).

${ }^{9}$ F. Hund, Z. Phys. 51, 1 (1928)

${ }^{10}$ M. Lagally and W. Franz, Vorlesungen über Vektorrechnung 7.Aufl. (Akadem. Buchgesellschaft, Geest \& Portig KG, Leipzig, 1964).

${ }^{11}$ G. Voll, Ph.D. thesis Universität Erlangen, 1987.

${ }^{12}$ A. Rogalsky, Diplomarbeit, Universität Erlangen, 1996.

${ }^{13}$ A. Hüller and D. M. Kroll, J. Chem. Phys. 63, 4495 (1975).

${ }^{14}$ A. Hüller and J. Raich, J. Chem. Phys. 71, 3851 (1979).

${ }^{15}$ Th. Brückel, W. Prandl, S. K. Vogt, and C. M. E. Zeyen, J. Phys. C 17, 4071 (1979).

${ }^{16}$ G. Voll and A. Hüller, Can. J. Chem. 66, 925 (1988).

${ }^{17}$ M. A. Neumann, W. Press, C. Nöldeke, B. Asmussen, M. Prager, and R. M. Ibbersen, J. Chem. Phys. 119, 1586 (2003).

${ }^{18}$ H. M. James and T. A. Keenan, J. Chem. Phys. 31, 12 (1959).

${ }^{19}$ W. Press, J. Chem. Phys. 56, 2597 (1972).

${ }^{20}$ W. Press and A. Hüller, Phys. Rev. Lett. 30, 1207 (1973).

${ }^{21}$ A. Hüller and W. Press, Phys. Rev. Lett. 29, 266 (1972).

${ }^{22}$ W. Press, A. Hüller, H. Stiller, W. Stirling, and R. Currat, Phys. Rev. Lett. 32, 1354 (1974).

${ }^{23}$ T. Yamamoto, Y. Kataoka, and K. Okada, J. Chem. Phys. 66, 2701 (1977).

${ }^{24}$ K. Maki, Y. Kataoka, and T. Yamamoto, J. Chem. Phys. 70, 655 (1979).

${ }^{25}$ M. Prager, W. Press, B. Asmussen, and J. Combet, J. Chem. Phys. 117, 5821 (2002) 